\title{
MIDPI
}

MOL2NET, International Conference Series on Multidisciplinary Sciences

sciforum,

\section{The Role of Biomedical Informatics in Exposome Research}

\author{
Guillermo H. Lopez-Campos ${ }^{a}$, Enrique Calderin ${ }^{b}$. \\ ${ }^{a}$ Wellcome-Wolfson Institute for Experimental Medicine, Faculty of Queen's University Belfast, \\ Belfast, $U K$ \\ ${ }^{b}$ Centre for Actuarial Sciences, Faculty of Business and Economics, The University of Melbourne, \\ Melbourne, Australia
}

\begin{abstract}
.
Biomedical informatics plays a major role in the advancement of exposome (defined as the complete set of exposures of an individual since conception) research providing tools and methods to effectively manage and analyse the information and data generated during these studies. Exposome research comprises a broad range of data sources some of which have been previously used in the biomedical informatics domain while some other such as those coming from the development of new sensors might be new. In this work we present three examples showing different aspects where biomedical informatics is either supporting or driving exposome research. Knowledge management and representation or potential use of microbiome data by life insurance industry as a mean to analyse risk represent examples of areas where biomedical informatics is supporting exposome research with its tools and methods. At the same time biomedical informatics could potentially lead exposome research in areas such as the study of the exposure to digital technologies and digital stimuli (the digital exposome).
\end{abstract}

\section{Biomedical informatics and the exposome}

The technological advances in the last decades have revolutionised many disciplines and have opened the doors for the development of new analytic methods and techniques enabling approaches that would have been difficult to imagine in the past. Genomics and other so-called "omics" approaches are one of the best examples of how these advances have influenced biomedical research and practice. When the original Human Genome Project was envisioned, it was considered the biomedical equivalent to the Apollo program. Three decades later, sequencing has become almost a standard technique and it is possible to sequence human individuals at large scales as in the 100,000 Genomes Project funded by genomics England (1) and the use of whole genome sequencing (WGS) and whole exome sequencing (WES) in clinical and medical practice is a reality.

At the same time, the advances in the development of new sensors and their integration in multiple consumer grade devices facilitating accessing to new sources of individual data some of which are relevant for health purposes. Probably the best example of these new data sources are physical activity trackers, which collect data and information related with the physical activity of the individuals and are 
integrated in most intelligent phones. Although physical activity is just the best known and popular of these new data sources the list extends also to many other areas such as air quality monitoring (2). Biomedical informatics has play a key role in the in the research and development of new tools and methods to efficiently manage, analyse and integrate all these new sources of data and information in the biomedical domain.

These advances and data rich context has fostered the development of what is currently known as precision medicine. A key element in precision medicine is the focus in the use of individual data and information (3). Currently these data may be originated and derived from different sources. These sources have been mostly grouped around the phenotypes the genotypes and in some cases the environmental factors. Nowadays these individual environmental factors are being grouped together around the concept of the exposome. The "Exposome" is defined as the complete set of exposures of an individual since conception (4). As it happened in the past with genome research and genomic data, the development of Exposome research and the exploitation of these new data in the context of human health require biomedical informatics research and support. The use of new data types and sources and their new potential applications represent a vast array of new challenges and opportunities for the development of new biomedical informatics tools and methods.

The exposome is a broad term that encompasses an extremely broad variety of data sources and data types some of which are new for biomedical informatics whereas some other might have already been of interest for biomedical informatics. Among the latter, microbiome information provides an example of data and information that has already been considered by biomedical informatics and now can be considered as part of the exposome.

Knowledge representation tools such as ontologies have been a successfully developed by biomedical informatics for many contexts and areas. Good examples of these ontologies are Gene Ontology (GO)

(5) or the Foundational Model of Anatomy (6) thanks to their widespread use and their relevance.

Exposome research could benefit as well from this kind of applications however and due to its relatively recent development it is unclear what could be (if any) the existing ontologies that could be better fit for their application in this area. In an analysis carried out in 2017 using a combination of inductive and deductive approaches on exposome related literature abstracts Kiossoglou et al. identified 17 ontologies containing classes that could be recognised in more than $50 \%$ of these abstracts (7). Not surprisingly, these were broad and general knowledge representation tools such as MESH, the NCI Thesaurus or SNOMED-CT.

An interesting area where biomedical informatics can contribute to the development of exposome research is what has been coined as the "digital exposome"(8). The digital exposome is a recent concept that acts as the digital counter part of the exposome. If the exposome is defined as the series of exposures of an individual, in an increasingly digitalized world the underlying idea of the digital exposome is to encompass the digital experiences of an individual. There is an increasing body of evidence in the literature linking online and digital experiences with health applications or conditions. Therefore it seems natural that biomedical informatics should play a leading role as a driving force in the support and development of this research.

Finally, the broad range of topics covered by exposome research includes the microbiome and who this dynamic element influences human health. Traditionally microbiome analyses have been related with translational bioinformatics as they heavily relay in molecular biology approaches.

Simultaneously the increasing knowledge about the roles of the microbiome in human health has related with a range of pathologies $(9,10)$ and even with longevity $(11)$. This has opened the doors for 
biomedical informatics to interact with other disciplines as actuarial sciences that are increasingly interested in the accessing and using different sorts of new biomedical data. In this scenario biomedical informatics would play a key role gathering, analysing and selecting the data that could be used later on by insurers.

\section{Acknowledgements}

Part of the work presented here was funded by Fundación MAPFRE, 2017 Ignacio Hernando De Larramendi Research Grants

\section{References}

1. Turnbull C. Introducing whole-genome sequencing into routine cancer care: the Genomics England 100000 Genomes Project. Ann Oncol. 2018;29(4):784-7.

2. Morawska L, Thai PK, Liu X, Asumadu-Sakyi A, Ayoko G, Bartonova A, et al. Applications of low-cost sensing technologies for air quality monitoring and exposure assessment: How far have they gone? Environ Int. 2018;116:286-99.

3. Lander ES. Cutting the Gordian helix--regulating genomic testing in the era of precision medicine. N Engl J Med. 2015;372(13):1185-6.

4. Wild CP. Complementing the genome with an "exposome": the outstanding challenge of environmental exposure measurement in molecular epidemiology. Cancer Epidemiol Biomarkers Prev. 2005;14(8):1847-50.

5. The Gene Ontology C. The Gene Ontology Resource: 20 years and still GOing strong. Nucleic Acids Res. 2018.

6. Rosse C, Mejino JL, Jr. A reference ontology for biomedical informatics: the Foundational Model of Anatomy. J Biomed Inform. 2003;36(6):478-500.

7. Kiossoglou P, Borda A, Gray K, Martin-Sanchez F, Verspoor K, Lopez-Campos G.

Characterising the Scope of Exposome Research: A Generalisable Approach. Stud Health Technol Inform. 2017;245:457-61.

8. Lopez-Campos G, Merolli M, Martin-Sanchez F. Biomedical Informatics and the Digital Component of the Exposome. Stud Health Technol Inform. 2017;245:496-500.

9. Kho ZY, Lal SK. The Human Gut Microbiome - A Potential Controller of Wellness and Disease. Front Microbiol. 2018;9:1835.

10. Pflughoeft KJ, Versalovic J. Human microbiome in health and disease. Annu Rev Pathol. 2012;7:99-122.

11. Dato S, Rose G, Crocco P, Monti D, Garagnani P, Franceschi C, et al. The genetics of human longevity: an intricacy of genes, environment, culture and microbiome. Mech Ageing Dev.

2017;165(Pt B):147-55. 